Check for updates

Cite this: RSC Adv., 2018, 8, 32886

\title{
Template-free synthesis of three dimensional porous boron nitride nanosheets for efficient water cleaning $\dagger$
}

\author{
Jie Li, ${ }^{\star a b}$ Shi He, ${ }^{a}$ Rui Li, ${ }^{\text {ab }}$ Wei Dai, ${ }^{\text {ab }}$ Junhui Tao, ${ }^{\text {ab }}$ Chuanhui Wang, ${ }^{\text {ab Junyi Liu, }}$ \\ Tian Wu (D) ${ }^{* a b}$ and Chengchun Tang (D) ${ }^{c}$
}

Preparation of efficient and reusable adsorption materials for water treatment and purification is still remarkably challenging. In this paper, three dimensional porous boron nitride nanosheets (3D porous BNNSs) with high chemical stability and excellent adsorption capacity for organic dyes have been successfully synthesized through a template-free route. The 3D porous BNNSs consist of uniform nanosheets with average diameter of about $200 \mathrm{~nm}$ and thickness of about $3 \mathrm{~nm}$. The adsorption conditions have been optimized by varying the experimental parameters such as initial dye concentration, solution $\mathrm{pH}$, contact time, etc. As expected, the 3D porous BNNSs exhibit superior adsorption activity toward methylene blue (MB) in aqueous solution: more than $95.3 \%$ of the dye can be removed within 5 min compared with the adsorption efficiency of $10 \%$ for conventional activated carbon and $67.5 \%$ for the $3 \mathrm{D}$ porous BNNSs reported previously at $\mathrm{pH} 8.0$ and $30{ }^{\circ} \mathrm{C}$. The unique $3 \mathrm{D}$ structure and high density adsorption sites are believed to play a key role in the efficient removal performance. Moreover, about $94.5 \%$ of the starting adsorption capacity is maintained after ten adsorptionregeneration cycles. With the high adsorption efficiency and reusability performance, the 3D porous BNNSs are suitable for water cleaning and meet the requirement of mass production.

Received 31st July 2018

Accepted 18th September 2018

DOI: 10.1039/c8ra06445h

rsc.li/rsc-advances
3D-BNNS networks, ${ }^{9}$ porous microsponges ${ }^{10}$ and ultralight boron nitride aerogels. ${ }^{11}$

Today, rapidly changing technologies, industrial products and practices generate waste and toxic pollutants that if improperly managed, have threatened human health and the environment. ${ }^{12}$ Taking into consideration the volume of discharge and effluent composition, textile industries are assessed as high polluters. Considerable attention has been paid to adsorption technology as an efficient and economical method of removing organic dyes from textile wastewater effluents. Owing to their high specific surface area, peculiar B-N bond polarity, and good thermal and chemical stability, the porous BN materials are the potential competitive candidates for the organic pollutions removal. ${ }^{13-17}$ To satisfy the requirement for practical applications, the 3D BN nanostructures have aroused continuous research interest because of combining the adsorption property of $\mathrm{BN}$ materials and characteristic of 3D nanostructures. Up to now, various formulations of $3 \mathrm{D}$ BN nanostructures have been explored and they have been used as adsorbents for the removal of organic dyes. Nanosheetstructured boron nitride spheres prepared by Liu et al. have shown excellent performance in wastewater treatment. ${ }^{18}$ Liu et al. have successfully synthesized functional 3D BN architecture for removal of dyes from water..$^{19}$ Xue $e t a l$. have fabricated boron nitride foam-like porous monoliths for efficient and fast removal of rhodamine B from the wastewater. ${ }^{5}$ The 3D white 
grapheme foam has been prepared by Zhao et al. that exhibits quick adsorption rate and high adsorption capacity for methylene blue. ${ }^{20}$

Herein, we report a template-free and catalyst-free route by which the $3 \mathrm{D}$ porous BNNSs with high thermal stability can be synthesized at large scale. The typical synthesis process consists of the formation of the precursor and the pyrolysis reaction in an ammonia flow. The as-grown 3D porous BNNSs with average diameters of about $200 \mathrm{~nm}$ and thicknesses of about $3 \mathrm{~nm}$ consist of an interconnected flexible network of BN nanosheets. Substantial experiments demonstrate that the 3D porous BNNSs possess high adsorption rate and capacity for organic dyes in the aqueous solution due to the combination of three dimensional $\mathrm{BN}$ structures and rich adsorption sites. The removal capacity of the $3 \mathrm{D}$ porous BNNSs is measured to be $413.3 \mathrm{mg} \mathrm{g}^{-1}$ for $\mathrm{MB}$ at $30{ }^{\circ} \mathrm{C}$ and $\mathrm{pH}$ 8. Moreover, after simple high temperature treatment, these used $3 \mathrm{D}$ porous BNNSs can be regenerated and used repeatedly with a negligible capacity loss (about 5.5\% of adsorption efficiency loses even after ten cycles). All these features make the 3D porous BNNSs suitable for water cleaning, indicate its potentiality in a wide range of applications including energy storage and catalytic support.

\section{Experimental}

\section{Synthesis}

All chemical reagents were used directly without further purification as starting materials. The synthesis process is briefly described as follows. Firstly, $0.05 \mathrm{~mol}$ of $\mathrm{B}_{2} \mathrm{O}_{3}$ was added into 0.1 mol of ethylene diamine solution, then the solution was mixed under vigorous stirring for $1 \mathrm{~h}$ to get a homogenous solution. Secondly, the homogenous mixture was placed into a three-necked round-bottomed flask and treated at $280{ }^{\circ} \mathrm{C}$ for $1 \mathrm{~h}$, then the as-prepared sample was naturally cooled to room temperature to get yellow colloform, a novel precursor. Finally, the as-prepared precursor was pretreated at $500{ }^{\circ} \mathrm{C}$ for $1 \mathrm{~h}$, and then the temperature was slowly increased to $1300{ }^{\circ} \mathrm{C}$ at a heating rate of $4{ }^{\circ} \mathrm{C} \mathrm{min}^{-1}$ to keep for another $2 \mathrm{~h}$ to obtained the 3D porous BNNSs. All reactions were carried out in an ammonia flow rate of $200 \mathrm{~mL} \mathrm{~min}^{-1}$.

\section{Characterization}

The phase of the $3 \mathrm{D}$ porous BNNSs were identified by X-ray powder diffraction (XRD, BRUKER D8 FOCUS). Fourier Transformation Infrared spectrometer (FTIR, Nicolet 7100) was used to feature the chemical compositions and bond characters. The morphology was examined by using field emission scanning electron microscopy (SEM, HITACHI S-4800) and transmission electron microscopy (TEM, Philips Tecnai F20) with an acceleration voltage of $200 \mathrm{kV}$. Thermogravimetry (TG) was measured at a controllable temperature ranging from room temperature to $1100{ }^{\circ} \mathrm{C}$ at a heating rate of $10{ }^{\circ} \mathrm{C} \mathrm{min}^{-1}$ under air flow on a SDTQ-600 thermal analyzer. The nitrogen adsorptiondesorption isotherm was measured at $77 \mathrm{~K}$ on an AutoSorb iQ-C TCD analyzer. The specific surface area was calculated by using
Brunauer-Emmett-Teller (BET) equation (eqn (1)) in the relative pressure ranging from 0.01 to 0.3 .

$$
\frac{1}{\left(W\left(\left(P_{0} / P\right)-1\right)\right)}=\frac{1}{W_{\mathrm{m}}}+\frac{C-1}{W_{\mathrm{m}}}\left(\frac{P}{P_{0}}\right)
$$

where $W$ is the weight of gas adsorbed at a relative pressure, $P_{0}$ is the saturated vapor pressure of the adsorbent, $P / P_{0}$ is the relative pressure, and $W_{\mathrm{m}}$ is weight of adsorbate constituting a monolayer of surface coverage. The term $C$, the BET $C$ constant, is related to the energy of adsorption in the first adsorbed layer and consequently its value is an indication of the magnitude of the adsorbent/adsorbate interactions. Due to the broad pore size distribution, the non-local density functional theory (NLDFT) ${ }^{21}$ method was used to calculate the pore widths and pore size distribution (ASiQwin software). In detail, a set of isotherms calculated for a set of pore sizes in a given range for a given adsorption constitutes the model database. Such a set of isotherms, called a kernel, is the basis for the pore size analysis by Density Functional Theory (DFT). The calculation of the pore size distribution is based on a solution of the generalized adsorption isotherm equation, which correlates the kernel of theoretical adsorption/desorption isotherms with the experimental sorption isotherm. In addition, the slit-shaped pores of the samples result from the aggregate of plate-like particles, these results are consistent with the NLDFT-N2-carbon equilibrium transition kernel at $77 \mathrm{k}$ based on a slit-pore model (ASiQwin software). The total pore volume is derived from the amount of vapor adsorbed at a relative pressure $P / P_{0}$ of 0.95 , by assuming that the pores are then filled with liquid adsorbate. The limiting adsorption can be identified reliably with the total pore volume assuming careful temperature control of the sample. The volume of nitrogen adsorbed ( $\left.V_{\text {ads }}\right)$ can be converted to the volume of liquid nitrogen $\left(V_{\text {liq }}\right)$ contained in the pores using eqn $\mathrm{S} 1$ (ESI $\dagger$ ). The $\mathrm{pH}$ of the solution was measured by $\mathrm{pH}$ meter (PHS-25, Hangzhou). The zeta potential was measured on an electric and particle analyzer (ELSZ-2000 ZS) at room temperature, as a function of $\mathrm{pH}$. The zeta potential measurements are performed using variable amounts of $0.1 \mathrm{M}$ $\mathrm{NaOH}$ and $0.1 \mathrm{M} \mathrm{HCl}$ to adjust the $\mathrm{pH}$ of the samples. A double beam UV/Vis spectrophotometer (HITACHI, U-3900H) was employed to measure the concentration of organic dyes in the aqueous solution.

\section{Batch adsorption experiments}

Batch adsorption experiments were undertaken in $100 \mathrm{~mL}$ flasks. To acquire the solution of organic dyes, methylene blue (MB) was dissolved in deionized water and then diluted to the required concentration before use. The $\mathrm{pH}$ of initial dye aqueous solution were adjusted to the required values by adding either $0.05 \mathrm{M} \mathrm{HCl}$ or $0.01 \mathrm{M} \mathrm{NaOH}$ solution. Buffer solution of potassium phosphate $(1 \mathrm{mM})$ was added to the dye solution to enable better control of the $\mathrm{pH}$ on the basis of the acid-base characteristic of the dyes. To investigate the influence of contact time, $50 \mathrm{mg}$ of the 3D porous BNNSs was brought into $100 \mathrm{~mL}$ of dye aqueous solution with a desired concentration and $\mathrm{pH}$ under continuously stirring at a constant speed of $150 \mathrm{rpm}$. The 
effect of $\mathrm{pH}$ on the adsorption capacity of the 3D porous BNNSs for $\mathrm{MB}$ was investigated in the $\mathrm{pH}$ range of 3-9. The amount of dye adsorbed onto the $3 \mathrm{D}$ porous BNNSs was calculated by a mass balance. The adsorption properties were measured at different time intervals at the optimized temperature and solution $\mathrm{pH}$. For comparison, the adsorption performances of the porous 3D BNNSs for methyl orange (MO) were also measured.

The pollutant removal percentage of the 3D porous BNNSs was calculated according to the following equation (eqn (2)):

$$
\eta(\%)=\left(C_{0}-C_{\mathrm{e}}\right) 100 / C_{0}
$$

where $C_{0}$ and $C_{\mathrm{e}}\left(\mathrm{mg} \mathrm{L}^{-1}\right)$ are the initial and equilibrium dye concentration, respectively. $\eta$ is the pollutant removal percentage of the pollutants.

The adsorption isotherm is fitted (correlation coefficients, $R^{2}$ $>0.99$ ) by using the following Langmuir adsorption model (eqn (3)):

$$
q_{\mathrm{e}}=q_{\mathrm{m}} K C_{\mathrm{e}} /\left(1+K C_{\mathrm{e}}\right)
$$

where $q_{\mathrm{e}}\left(\mathrm{mg} \mathrm{g}^{-1}\right)$ is the adsorbed amount of pollutant on the equilibrium concentration, $q_{\mathrm{m}}\left(\mathrm{mg} \mathrm{g}^{-1}\right)$ is the maximum adsorption capacity corresponding to complete monolayer covering on the adsorbents, $C_{\mathrm{e}}\left(\mathrm{mg} \mathrm{L}^{-1}\right)$ is the equilibrium concentration in solution, and $K\left(\mathrm{~L} \mathrm{mg}^{-1}\right)$ is the equilibrium constant related to the free energy of adsorption.

\section{Regeneration and reusability}

The regeneration of the dye-containing 3D porous BNNSs was carried out by annealing at $400{ }^{\circ} \mathrm{C}$ in air for $1 \mathrm{~h}$.

\section{Results and discussion}

\section{Characterization of the adsorbents}

To study the crystal structure of the as-prepared 3D porous BNNSs, the powder XRD pattern is investigated. The typical XRD pattern is shown in Fig. 1a, in which the diffraction peak at $26.5^{\circ}$ can be indexed as (002) lattice plane, indicating towards the hexagonal type of crystal structure in the hexagonal boron nitride (h-BN) (JCPDS card no. 34-0421)..$^{22,23}$ The peaks at $42.1^{\circ}$, $54.8^{\circ}$ and $76.4^{\circ}$ can also be indexed as the (100), (004), and (110) planes of h-BN, respectively. The sharpness of the main peaks and the appearance of the peak at $76.4^{\circ}$ corresponding to the interplanar order (110) plane confirm that the 3D porous BNNSs possess higher degree of crystallinity than that of porous $\mathrm{BN}$ or activated BN. ${ }^{\mathbf{1 4 2 4}}$ The $3 \mathrm{D}$ porous BNNSs with high crystallinity makes themselves possess good stability in the adsorption process, which is consistent with the result of TG measurement (please see the following section).

The structural information and surface chemical properties of 3D porous BNNSs are illustrated by the FTIR spectrum in Fig. 1b. The two strong vibrations at around 1400 and $800 \mathrm{~cm}^{-1}$ in the FTIR spectrum attribute towards the in-plane stretching vibration of $\mathrm{sp}^{2}$-bonded $\mathrm{B}-\mathrm{N}$ and the out-of-plane bending vibration of $\mathrm{sp}^{2}$-bonded $\mathrm{B}-\mathrm{N}-\mathrm{B}$, respectively. ${ }^{25}$ Apart from the
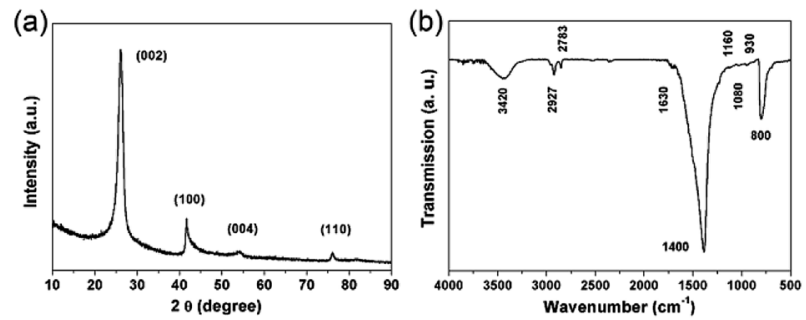

Fig. 1 (a) Powder XRD pattern and (b) typical FTIR spectrum of the 3D porous BNNSs.

intrinsic peaks of h-BN, several weak peaks are also observed, including $\mathrm{CH} \quad\left(\sim 2927 \mathrm{~cm}^{-1}\right), \quad-\mathrm{CH}_{3} \quad\left(\sim 2783 \mathrm{~cm}^{-1}\right), \quad \mathrm{CO}$ $\left(\sim 1630 \mathrm{~cm}^{-1}\right), \mathrm{B}-\mathrm{N}-\mathrm{O}\left(\sim 1160 \mathrm{~cm}^{-1}\right)$, CO $\left(\sim 1080 \mathrm{~cm}^{-1}\right)$, and $\mathrm{B}-\mathrm{N}-\mathrm{O}\left(\sim 930 \mathrm{~cm}^{-1}\right) .{ }^{26}$ It is worth noting that the extra peak centered at $3420 \mathrm{~cm}^{-1}$ can be ascribed to the hydroxyl group bonding to B atom or absorbed moisture. The FTIR analyses indicate that the $3 \mathrm{D}$ porous BNNSs possess abundant hydroxyl and surface organic groups, which are conducive to enhanced removal performance of the $3 \mathrm{D}$ porous BNNSs for organic dyes.

From the SEM observation in Fig. 2a, a foam-like morphology in the overall can be certified as the representative of the $3 \mathrm{D}$ porous BNNSs, which are prepared by a templatefree synthetic method at $1300{ }^{\circ} \mathrm{C}$. A photo of the sample is displayed in Fig. S1 (ESI $\dagger$ ). The 3D porous BNNSs consist of BN nanosheets, and exhibit porous, fluffy and corrugated structures. The high-magnification SEM image (Fig. 2b) illustrates that the average diameters of the $\mathrm{BN}$ nanosheets assembling the foam-like 3D porous BNNSs are about $200 \mathrm{~nm}$, and further reveal that the $3 \mathrm{D}$ porous BNNSs comprise a $3 \mathrm{D}$ porous structure. It is noticed that the pore volume of the $3 \mathrm{D}$ porous BNNSs is attributed to the aggregation of the $\mathrm{BN}$ nanosheets rather than their intraplate holes, which is further confirmed by the results from the analysis of the pore size distribution (please see the following section). The TEM images provide an insight
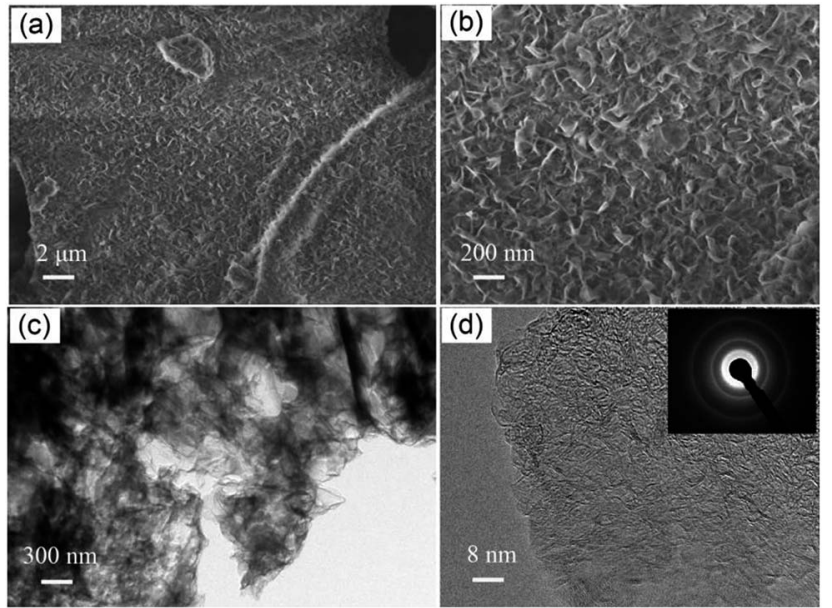

Fig. 2 (a) Typical SEM image of the 3D porous BNNSs. (b) The corresponding high-magnification SEM image. (c) TEM image of the porous 3D BNNSs. (d) The corresponding high-magnification TEM image (inset is the selected area electron diffraction pattern). 
vision of structure, morphology and nanosheet size of the 3D porous BNNSs. The TEM image reveals that the 3D porous BNNSs are consisted of interconnected wrinkled layers, as displayed in Fig. 2c. The corresponding high-magnification TEM image in Fig. 2d further indicates the wrinkled layer structures, and also depicts the presence of specific lattice planes. The atomic layers of the $\mathrm{BN}$ nanosheets rang from 4 to 10 layers. The selected area electron diffraction (SAED) pattern (Fig. 2d, inset) confirms the presence of crystalline nature corresponding to the (002) and (100) diffraction rings related to h-BN, which is also accordance with the above-mentioned XRD observation. ${ }^{27} \mathrm{~A}$ further investigation of the outer surface of the nanosheets reveals that numerous surface defects are introduced in the surface of the 3D porous BNNSs. The exposed BN edges with rich surface defects can provide excellent adsorption reactivity of the 3D porous BNNSs for organic dyes in the aqueous solution due to the high density structural defects offering strong binding sites.

The specific surface area, pore volume and pore size distribution play the significant roles during the adsorption process. The outgassing of the $3 \mathrm{D}$ porous BNNSs was carried at $300{ }^{\circ} \mathrm{C}$ for $3 \mathrm{~h}$ prior to nitrogen adsorption/desorption measurement. The nitrogen adsorption/desorption isotherm is conducted to determine the specific surface area and the pore size distribution of the 3D porous BNNSs. As displayed in Fig. 3a, the obtained isotherm is a characteristic of type-II isotherm according to the IUPAC nomenclature, ${ }^{\mathbf{2 8}}$ and exhibits a $\mathrm{H} 3$ type broad hysteresis loop at a relative pressure between about 0.6 and 1.0. It is depicted that the $3 \mathrm{D}$ porous BNNSs possess predominant mesoporous structures with the slit-shaped pores, which result from the accretion of the BN nanosheets. The specific surface area of $474 \mathrm{~m}^{2} \mathrm{~g}^{-1}$ can be calculated by using the BET model. ${ }^{29}$ The broad pore diameters ranging from 3.8 to $29 \mathrm{~nm}$ have the main characteristic pore size of about $15 \mathrm{~nm}$ calculated by using the NLDFT method, ${ }^{30,31}$ as shown in Fig. $3 \mathrm{~b}$. On the basis of the isotherm profile, the pore volume is calculated to be $0.77 \mathrm{~cm}^{3}$ $\mathrm{g}^{-1}$.

Zeta potential, revealing the type and value of the surface charge, plays a significant role to control the adsorption capacity and rate of the $3 \mathrm{D}$ porous BNNSs for the different organic dyes. For the $3 \mathrm{D}$ porous BNNSs, the medium is kept water at a range of $\mathrm{pH}$ (ranging from 2 to 8). A constant voltage $(3.4 \mathrm{~V})$ is applied to the electrodes placed in the dispersion medium. The conductivities of the $3 \mathrm{D}$ porous BNNSs are
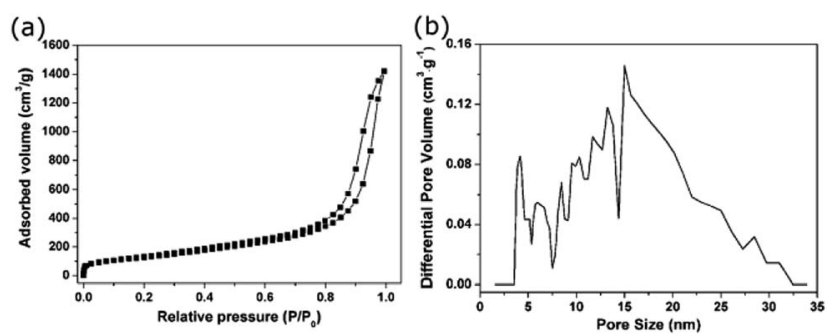

Fig. 3 (a) Nitrogen adsorption/desorption isotherm of the 3D porous BNNSs, (b) the corresponding pore size distributions obtained by DFT method.

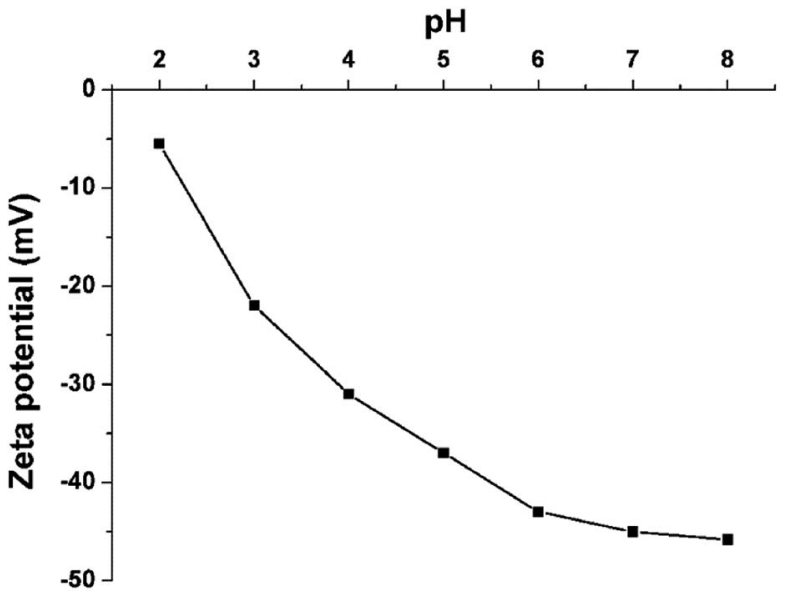

Fig. 4 Zeta potential vs. pH values of the 3D porous BNNSs.

measured at different $\mathrm{pH}$, and the corresponding zeta potential values are evaluated to be -5 to $-45.8 \mathrm{mV}$ at $\mathrm{pH}$ (ranging from 2 to 8 ), as shown in Fig. 4 . The negative values of zeta potential confirm that the $3 \mathrm{D}$ porous BNNSs possess an overall negative surface charge even at above $\mathrm{pH} 2$. Such negative surface charge is mainly attributed to the hydroxyl and amino groups on the surface and numerous structural defects. ${ }^{5}$ In addition, the zeta potential of the $3 \mathrm{D}$ porous BNNSs become less negative with an decrease in $\mathrm{pH}$ of the aqueous solution, as a result of the protonation of the $-\mathrm{OH} /-\mathrm{NH}_{2}$ on the surface. ${ }^{17}$ Relatively enormous negative charges at high $\mathrm{pH}$ lead to improved electrostatic attraction between the 3D porous BNNSs and the cationic dye, which facilitates the adsorption of MB.

The high thermostability of the adsorbent enhances their cost-effectiveness due to long-term reusability. Fig. 5 displays the TG curve of the as-prepared 3D porous BNNSs measured in an air flow. From TG observation, the serious oxidation of the porous 3D BNNSs happens at about $930{ }^{\circ} \mathrm{C}$, which is much higher than that of their analogue, the activated carbon. ${ }^{32}$ The higher thermostability and resistance to oxidation enables

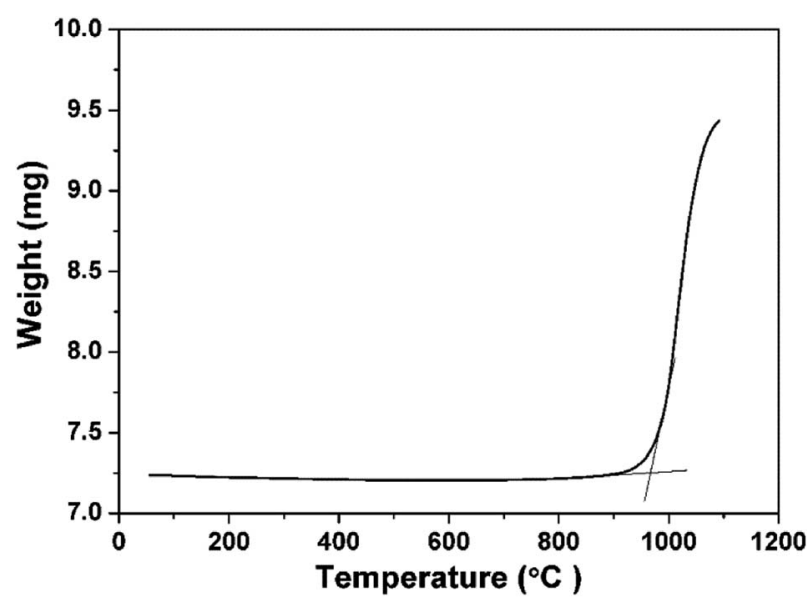

Fig. 5 Thermogravimetry (TG) curve of the 3D porous BNNSs performed in an air flow. Temperature: $1100{ }^{\circ} \mathrm{C}$; step: $10^{\circ} \mathrm{C} \mathrm{min}^{-1}$. 
better stability of the sorbent in adsorption and regeneration process, which is consistent with the result of XRD measurement. This result also suggests that the regeneration of the dyecontaining 3D porous BNNSs is carried out by a simple high temperature treatment. It is believed that the 3D porous BNNSs can also be a promising candidate for high-temperature applications in the air.

\section{Adsorption studies}

MB and MO as the pollutants are employed to study the adsorption properties and demonstrate the potential applicability of the $3 \mathrm{D}$ porous BNNSs in wastewater treatment. In addition, the effects of the experimental parameters, such as solution $\mathrm{pH}$, concentrations and contact time on removal efficiency of the 3D porous BNNSs for the organic dyes are investigated to achieve high adsorption capacity and rate in details.

The solution $\mathrm{pH}$ plays an important role in the whole adsorption process and particularly on the adsorption capacity. The variations of $\mathrm{MB}$ and $\mathrm{MO}$ adsorption on the $3 \mathrm{D}$ porous BNNSs over a broad range of $\mathrm{pH}$ (ranging from 3 to 9) are shown in Fig. 6. It is known that ionic dyes upon dissolution release coloured dye anions/cations into the solution. It is obvious that the different trends of adsorption are followed by both dyes due to the types of charge on their surface. As shown in Fig. 6, the equilibrium adsorption capacities of the 3D porous BNNSs for MB increase with increasing solution $\mathrm{pH}$, the adsorption mechanism for organic compounds is similar to that of the highly water-soluble and porous BN materials reported previously. ${ }^{33}$ Specially, these equilibrium adsorption capacity has more significant enhancement in the adsorption process as the solution $\mathrm{pH}$ is reached at 8 rather than at 7 or 9 . The maximum adsorption percentage is up to $98.3 \%$ at $\mathrm{pH} 8$, which is consistent with the zeta potential measurement. This can be attributed to the electrostatic attraction between the negatively charged surface of the $3 \mathrm{D}$ porous BNNSs and the cationic dye (MB). Decreasing in competition between $\mathrm{H}^{+}$and positively charge dye leads to more availability of adsorption sites with the

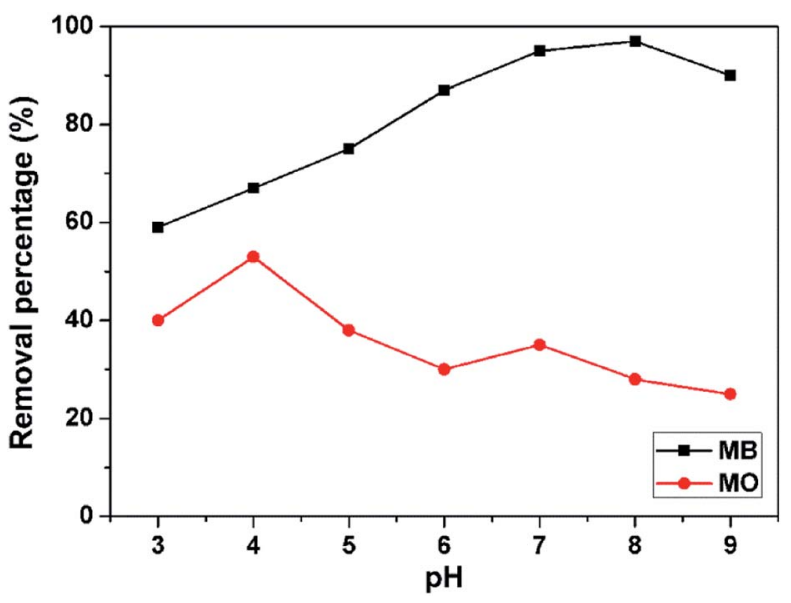

Fig. 6 Effect of solution $\mathrm{pH}$ on the equilibrium removal percentage of MB (mass of adsorbent: $50 \mathrm{mg}$, contact time: $180 \mathrm{~min}$, adsorption temperature: $30{ }^{\circ} \mathrm{C}$, dye concentration: $200 \mathrm{mg} \mathrm{L}^{-1}$ ).

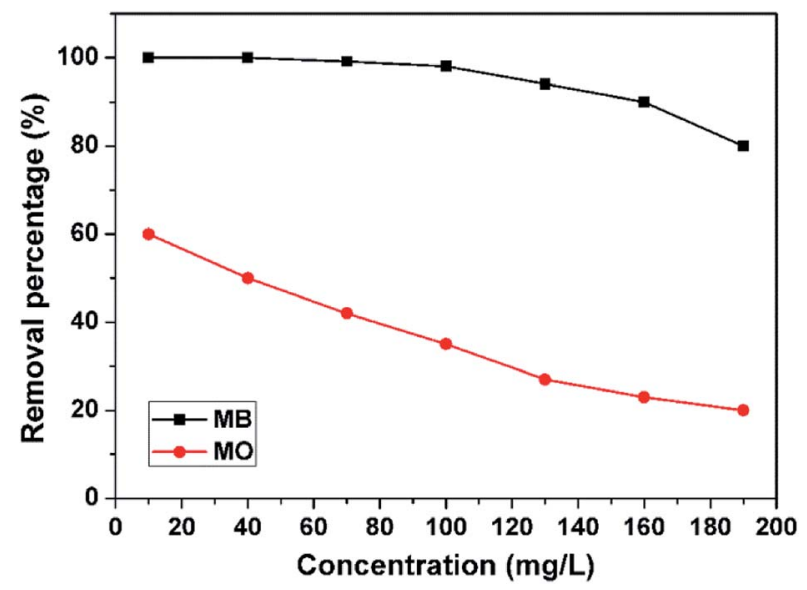

Fig. 7 Effect of initial dye concentrations on adsorption of $M B$ (mass of adsorbent: $50 \mathrm{mg}$, solution $\mathrm{pH}$ : 8, contact time: $180 \mathrm{~min}$, adsorption temperature: $30^{\circ} \mathrm{C}$ ).

increase of solution $\mathrm{pH}^{34}$ By contraries, the adsorption efficiency of the $3 \mathrm{D}$ porous BNNSs for the anionic dyes such as MO is enhanced with the decrease of $\mathrm{pH}$ and reaches the maximum adsorption percentage at $\mathrm{pH}$ 4. This result suggests that the reduction of negative charge on the surface of the $3 \mathrm{D}$ porous BNNSs is cause of neutralizing with $\mathrm{H}^{+}$. Different from the adsorption of cationic dye (MB), the electrostatic repulsion between the $3 \mathrm{D}$ porous BNNSs with negative charge and anionic dye (MO) results in decreasing the adsorption capacity. Therefore, the adsorption of these charged dyes onto the surface of the $3 \mathrm{D}$ porous BNNSs is primarily influenced by the surface charge on the adsorbent which is in turn influenced by the solution $\mathrm{pH}$.

The MB adsorption of the 3D porous BNNSs as a function of the initial pollution concentrations is depicted in Fig. 7. The initial pollution concentrations range from $5 \mathrm{mg} \mathrm{L}^{-1}$ to $190 \mathrm{mg} \mathrm{\textrm {L } ^ { - 1 }}$ while other experimental parameters, such as solution $\mathrm{pH}$, contact time, are kept constant. The removal percentages of $\mathrm{MB}$ are investigated to be decreased with an increase in the initial concentrations due to the reduction in the effective adsorption sites of the $3 \mathrm{D}$ porous BNNSs. The adsorption of $\mathrm{MO}$ on the $3 \mathrm{D}$ porous BNNSs is also displayed in Fig. 7 for comparison. Under the same experimental conditions, the removal percentage of MB is much higher than that of MO. It is believed that the high density hydroxyl and amino groups and surface defects on the $3 \mathrm{D}$ porous BNNSs provide a large number of active sites for the adsorption of the cationic dye (MB) rather than the anionic dye (MO). ${ }^{35}$

The contact time is very important parameter to evaluate the rate of adsorption. As shown in Fig. 8, the removal rate of the 3D porous BNNSS for MB is very fast, especially in the first $5 \mathrm{~min}$. About $95.3 \mathrm{wt} \%$ of $\mathrm{MB}$ in the aqueous solution is removed in the first $5 \mathrm{~min}$ in comparison with the adsorption efficiency of $10 \mathrm{wt} \%$ for conventional activated carbon and $67.5 \mathrm{wt} \%$ of the 3D porous BNNSs reported previously (Fig. S2, ESI $\dagger$ ), ${ }^{7}$ and the removal percentage of $\mathrm{MB}$ increase to $99 \mathrm{wt} \%$ at $20 \mathrm{~min}$. The equilibrium adsorption capacity (99.5 wt\%) for $\mathrm{MB}$ on the $3 \mathrm{D}$ BNNSs quickly reach at $30 \mathrm{~min}$. On the contrary, only 


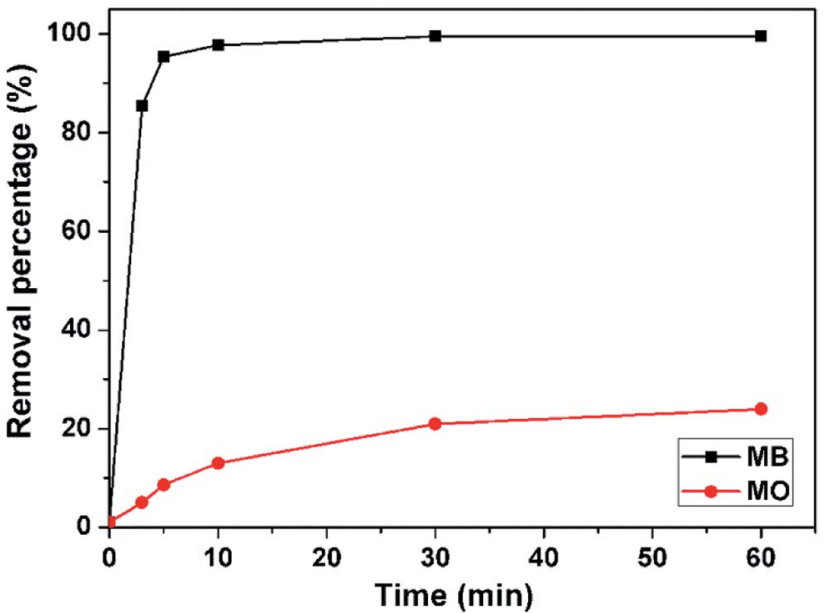

Fig. 8 Effect of contact time on adsorption of MB (mass of adsorbent: $50 \mathrm{mg}$, solution $\mathrm{pH}: 8$, dye concentration: $100 \mathrm{mg} \mathrm{L}^{-1}$, adsorption temperature: $30^{\circ} \mathrm{C}$ ).

$\sim 28.3 \mathrm{wt} \%$ of $\mathrm{MO}$ in the aqueous solution is removed within $60 \mathrm{~min}$. This suggests that the overall negative charges of the 3D porous BNNSs are favorable for enhancing attractive forces between the unsaturated adsorbent surface and unabsorbed cationic dye (MB) rather than anionic dye (MO) molecules in the aqueous solution.

The experimental data are applied to the Langmuir isotherm model to investigate the adsorption behaviors of $\mathrm{MB}$ on the 3D porous BNNSs, as displayed in Fig. 9. The adsorption isotherm indicates that Langmuir model has a good fit with the experimental data for the $3 \mathrm{D}$ porous BNNSs (correlation coefficients, $R^{2}>0.99$ ). This suggests that all sites are equal (equal enthalpies and energies). The maximum adsorption capacity of the 3D porous BNNSs calculated from the Langmuir equation is up to $413.3 \mathrm{mg} \mathrm{g}^{-1}$, which is higher than those of most nanomaterials reported previously. ${ }^{14,17,36-38}$ It is noticed that the removal capacity is also higher than most of the $\mathrm{BN}$ materials reported previously including porous $\mathrm{BNNSs}^{\mathbf{1 4}} \mathrm{BN}$ nanocarpets, ${ }^{\mathbf{1 6}}$

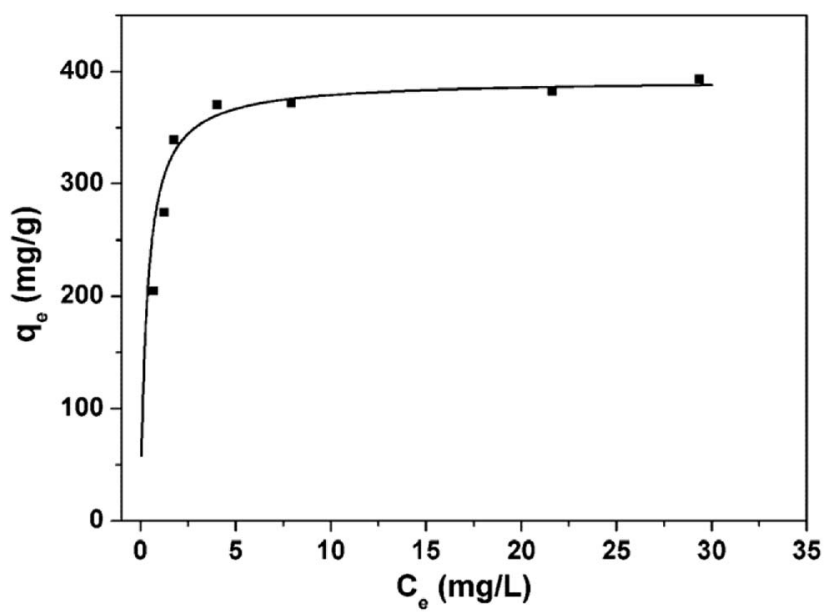

Fig. 9 Adsorption isotherm of $\mathrm{MB}$ on the 3D porous BNNSs (contact time: $180 \mathrm{~min}$, solution $\mathrm{pH}: 8$, temperature: $30^{\circ} \mathrm{C}$ ).

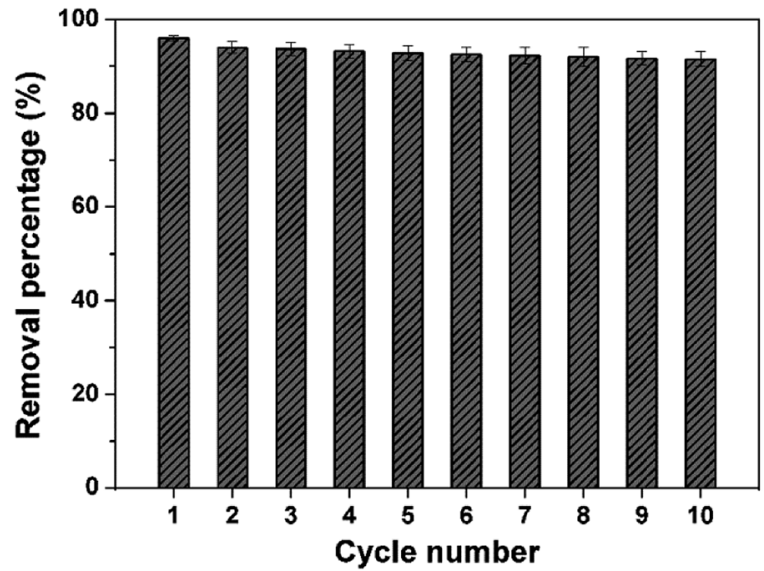

Fig. 10 Reusability of the 3D porous BNNSs regenerated by combustion.

activated $\mathrm{BN},{ }^{17} 3 \mathrm{D}$ BNNSs foam,${ }^{20} \mathrm{BN}$ hollow spheres, ${ }^{39}$ as listed in Table S1 (ESI $\dagger$ ). A very steep slope in the initial portion of the adsorption isotherm reveals high adsorption capacity and rate of the 3D porous BNNSs. The high efficiency of the 3D porous BNNSs for water cleaning can be attributed to unique $3 \mathrm{D}$ structure and high density adsorption sites.

The reliable recovery and recycling of the 3D porous BNNSs is very important for application in water cleaning. A hightemperature treatment in air is used to regenerate organic dyes-saturated 3D porous BNNSs. Batch of regeneration experiments shown in Fig. 10 reveal that about $94.5 \%$ of the starting adsorption capacity is retained even after ten adsorption-regeneration cycles, which is much higher than that of the conventional activated carbon (the most widely used material for water cleaning at present). Moreover, the removal efficiency has no obvious decrease with the increase of cycles. From the XRD and SEM observations (Fig. S3, ESI $\dagger$ ), one can see that the structure and morphology of the regenerated 3D porous BNNSs have no obvious change. The efficient reusability is mainly attributed to the high thermostability and oxidation resistance, which can enhance the cost-effectiveness of the $3 \mathrm{D}$ porous BNNSs.

\section{Conclusions}

In summary, the $3 \mathrm{D}$ porous BNNSs with high thermostability are successfully prepared by the template-free synthesis route without the use of any catalyst, which consist of the corrugated $\mathrm{BN}$ nanosheets. Solution $\mathrm{pH}$ plays very important role in the adsorption process on basis of the investigation of negative charge over the surface on the 3D porous BNNSs. The 3D porous BNNSs exhibit excellent adsorption capacity without any additives as high as $413.3 \mathrm{mg} \mathrm{L}^{-1}$ and adsorption rate (effectively eliminating $\sim 95.3 \mathrm{wt} \%$ within $5 \mathrm{~min}$ ) at $\mathrm{pH}$ 8. It also features good cycling performance due to high resistance to oxidation and good chemical inertness. Therefore, the 3D porous BNNSs are very promising candidates for application in water cleaning. 


\section{Conflicts of interest}

There are no conflicts to declare.

\section{Acknowledgements}

This work was supported by the Natural Science Foundation of Hubei Province (2017CFB581), the Science and Technology Research Program of the Education Department of Hubei Province (B2017210), Major Project of Technological Innovation (2016AAA038).

\section{Notes and references}

1 J. G. Alauzun, S. Ungureanu, N. Brun, S. Bernard and P. Miele, J. Mater. Chem., 2011, 21, 14025.

2 M. Rousseas, A. P. Goldstein, W. Mickelson, M. A. Worsley, L. Woo and A. Zettl, ACS Nano, 2013, 7, 8540.

3 J. Yin, X. Li, J. Zhou and W. Guo, Nano Lett., 2013, 13, 3232. 4 H. Wang, K. Sun, F. Tao, D. Stacchiola and Y. H. Hu, Angew. Chem., Int. Ed., 2013, 52, 9210.

5 Y. Xue, P. Dai, X. Jiang, X. Wang, C. Zhang, D. Tang, Q. Weng, X. Wang, A. Pakdel, C. Tang, Y. Bando and D. Golberg, J. Mater. Chem. A, 2016, 4, 1469.

6 T. Ohashi, Y. T. Wang and S. Shimada, J. Mater. Chem., 2010, 20, 5129.

7 J. Li, H. Jia, Y. Ding, H. Luo, S. Abbas, Z. Liu, L. Hu and C. Tang, Nanotechnology, 2015, 26, 475704.

8 G. Lian, X. Zhang, M. Tan, S. J. Zhang, D. L. Cui and Q. L. Wang, J. Mater. Chem., 2011, 21, 9201.

9 X. Zeng, Y. Yao, Z. Gong, F. Wang, R. Sun, J. Xu and C. Wong, Small, 2015, 11, 6205.

10 Q. Weng, X. Wang, Y. Bando and D. Golberg, Adv. Energy Mater., 2013, 4, 1.

11 Y. Song, B. Li, S. Yang, G. Ding, C. Zhang and X. Xie, Sci. Rep., 2014, 5, 10337.

12 E. Forgacs, T. Cserháti and G. Oros, Environ. Int., 2004, 30, 953.

13 Q. Li, T. Yang, Q. Yang, F. Wang, K. C. Chou and X. Hou, Ceram. Int., 2016, 42, 8754.

14 W. Lei, D. Portehault, D. Liu, S. Qin and Y. Chen, Nat. Commun., 2013, 4, 1777.

15 L. Xue, B. Lu, Z. Wu, C. Ge, P. Wang, R. Zhang and X. Zhang, Chem. Eng. J., 2014, 243, 494.

16 X. Zhang, G. Lian, S. Zhang, D. Cui and Q. Wang, CrystEngComm, 2012, 14, 4670.

17 J. Li, Y. Huang, Z. Liu, J. Zhang, X. Liu, H. Luo, Y. Ma, X. Xu, Y. Lu, J. Lin, J. Zuo and C. Tang, J. Mater. Chem. A, 2015, 3, 8185.
18 F. Liu, J. Yu, X. Ji and M. Qian, ACS Appl. Mater. Interfaces, 2015, 7, 1824.

19 D. Liu, W. Lei, S. Qin and Y. Chen, Sci. Rep., 2014, 4, 4453. 20 H. Zhao, X. Song and H. Zeng, NPG Asia Mater., 2015, 7, 1.

21 The Non-Local Density Functional Theory (NLDFT) methods used in the present manuscript correctly describe the local fluid structure near curved solid walls; adsorption isotherms in model pores are determined based on the intermolecular potentials of the fluid-fluid and solid-fluid interactions, the related explanation please read the references, R. Evans, U. M. B. Marconi and P. Tarzona, J. Chem. Soc., Faraday Trans. 2, 1986, 82, 1763; P. I. Ravikovitch, G. L. Haller and A. V. Neimark, $A d v$. Colloid Interface Sci., 1998, 203, 76-77.

22 C. Zhi, Y. Bando, C. Tang, D. Golberg, R. Xie and T. Sekigushi, Appl. Phys. Lett., 2005, 86, 213110.

23 R. T. Paine and C. K. Narula, Chem. Rev., 1990, 90, 73.

24 D. Golberg, Y. Bando, Y. Huang, T. Terao, M. Mitome, C. Tang and C. Zhi, ACS Nano, 2010, 4, 2979.

25 C. Zhi, Y. Bando, C. Tang, H. Kuwahara and D. Golberg, Adv. Mater., 2009, 21, 2889.

26 C. Tang, Y. Bando, Y. Huang, C. Zhi and D. Golberg, Adv. Funct. Mater., 2008, 18, 3653.

27 J. Li, P. Jin, W. Dai, C. Wang, R. Li, T. Wu and C. Tang, Mater. Chem. Phys., 2017, 196, 186.

28 K. S. W. Sing, Pure Appl. Chem., 1985, 57, 603.

29 S. Brunauer, P. H. Emmett and E. Teller, J. Am. Chem. Soc., 1938, 60, 309.

30 R. Evans and P. Tarazona, Phys. Rev. Lett., 1984, 52, 557.

31 S. Schlieger, J. Alauzun, F. Michaux, L. Vidal, J. Parmentier, C. Gervais, F. Babonneau, S. Bernard, P. Miele and J. B. Parra, Chem. Mater., 2012, 24, 88.

32 M. Sekar, V. Sakthi and S. Rengaraj, J. Colloid Interface Sci., 2004, 279, 307.

33 Q. Weng, B. Wang, X. Wang, N. Hanagata, X. Li, D. Liu, X. Wang, X. Jiang, Y. Bando and D. Golberg, ACS Nano, 2014, 8, 6123-6130.

34 E. N. E. Qada, S. J. Allen and G. M. Walker, Chem. Eng. J., 2008, 135, 174-184.

35 P. Singla, N. Goel, V. kumar and S. Singhal, Ceram. Int., 2015, 41, 10565.

36 S. Wang, Y. Boyjoo, A. Choueib and Z. H. Zhu, Water Res., 2005, 39, 129.

37 L. Zheng, Y. Su, L. Wang and Z. Jiang, Sep. Purif. Technol., 2009, 68, 244.

38 C. Kannan, K. Muthuraja and M. R. Devi, J. Hazard. Mater., 2013, 244-245, 10.

39 G. Lian, X. Zhang, S. Zhang, D. Liu, D. Cui and Q. Wang, Energy Environ. Sci., 2012, 5, 7072. 